\title{
Decline in macrolide resistance rates among Streptococcus pyogenes causing pharyngitis in children isolated in Italy
}

\author{
G. Gherardi ${ }^{1}$ - D. Petrelli ${ }^{2}$ M. C. Di Luca ${ }^{2,7}$. \\ F. Pimentel de Araujo ${ }^{1,8} \cdot$ P. Bernaschi $^{3} \cdot$ A. Repetto $^{4}$. \\ J. Bellesi ${ }^{5}$ L. A. Vitali ${ }^{6}$
}

Received: 12 May 2015 / Accepted: 13 May 2015 / Published online: 30 May 2015

(C) The Author(s) 2015. This article is published with open access at Springerlink.com

\begin{abstract}
Macrolides are often used to treat group A streptococcus (GAS) infections, but their resistance rates reached high proportions worldwide. The aim of the present study was to give an update on the characteristics and contemporary prevalence of macrolide-resistant pharyngeal GAS in Central Italy. A total of 592 isolates causing pharyngitis in children were collected in the period 2012-2013. Clonality was assessed by emm typing and pulsed-field gel electrophoresis (PFGE) for all macrolide-resistant strains and for selected susceptible isolates. Genetic determinants of resistance were screened by polymerase chain reaction (PCR). Forty-four GAS were erythromycin-resistant $(7.4 \%)$. Among them, $52.3 \%$ and $50 \%$ were clindamycin- and tetracycline-resistant,
\end{abstract}

G. Gherardi and D. Petrelli contributed equally to this work.

L. A. Vitali

luca.vitali@unicam.it

University Campus Bio-Medico, 00128 Roma, Italy

2 School of Biosciences and Veterinary Medicine, University of Camerino, 62032 Camerino, MC, Italy

3 Unit of Microbiology, Bambino Gesù Children's Hospital, Roma, Italy

4 Azienda Ospedaliera di Perugia, Struttura Complessa di Microbiologia, Santa Maria della Misericordia Hospital Perugia, Perugia, Italy

5 Azienda Ospedaliera di Macerata, Macerata, Italy

6 School of Pharmacy, Microbiology Unit, University of Camerino, via Gentile III da Varano, 62032 Camerino, MC, Italy

7 Present address: Department of Microbiology and Infection Control, University Hospital of North Norway, 9038 Tromsø, Norway

8 Present address: Department of Infectious, Parasitic and Immune-Mediated Diseases, Istituto Superiore di Sanità, Viale Regina Elena 299, 00161 Rome, Italy respectively. $\operatorname{erm}(\mathrm{B})$-positive isolates $(52.3 \%)$ expressed the constitutive $\mathrm{cMLS}_{\mathrm{B}}$ phenotype. mef(A) and its associated M phenotype were recorded in $40.9 \%$ of the cases. The remaining $\operatorname{erm}(\mathrm{A})$-positive isolates expressed the iMLS $_{\mathrm{B}}$ phenotype. Seventeen tetracycline-resistant isolates carried tet $(\mathrm{M})$ and five isolates carried tet $(\mathrm{O})$. Twenty-five emm types were found among all strains, with the predominance of emm types 12, 89, 1, and 4. Eleven emm types and 12 PFGE clusters characterized macrolide-resistant strains, with almost two-thirds belonging to emm 12, emm 4, and emm 11. Macrolidesusceptible and -resistant emm types 12, 89, 11, and 4 shared related PFGE profiles. There was a dramatic decline in macrolide resistance in Central Italy among pharyngeal GAS isolates in 2012-2013 when compared to previous studies from the same region $(p<0.05)$, although macrolide consumption remained stable over the past 15 years. We observed a decrease in the proportion of macrolide-resistant strains within emm types commonly associated with macrolide resistance in the past, namely emm 12,1 , and 89 .

\section{Introduction}

Streptococcus pyogenes, or Lancefield group A streptococcus (GAS), is an important pathogen implicated in a wide variety of human infections. The species is associated with both noninvasive diseases, such as acute pharyngitis, an infection for which it is the most common bacterial agent, and invasive infections, such as skin and soft-tissue infections, necrotizing fasciitis, bacteremia, sepsis, and toxic shock syndrome [1].

GAS remains sensitive to $\beta$-lactams, which is the drug class of choice in the treatment of most streptococcal infections because of its narrow spectrum of action and its efficacy in the prevention of post-streptococcal sequelae, such as 
rheumatic fever [1]. Macrolides have been recommended for patients allergic to $\beta$-lactams, and clindamycin is the preferred antibiotic in the treatment of patients with serious soft-tissue infections because of its ability to inhibit the production of several streptococcal virulence factors [1]. Resistance to erythromycin and related antibiotics has represented an important cause of concern $[2,3]$ and is mainly associated with two mechanisms. The first is expressed by mef genes, such as mef(A), encoding for an efflux pump, which confers resistance to 14 - and 15-membered ring macrolides and susceptibility to clindamycin (M phenotype) [4]. The second mechanism involves erm genes, including erm(A) [subclass erm(TR)] and $\operatorname{erm}(\mathrm{B})$, which encode methylases targeting 23S rRNA [5]. The modification is associated with a decreased binding of all macrolides, lincosamides, and streptogramin type B to their targets on the ribosomal RNA (MLS $\mathrm{B}_{\mathrm{B}}$ phenotype), and it can be either induced (iMLS $S_{B}$ phenotype) or constitutive ( $\mathrm{cMLS}_{\mathrm{B}}$ phenotype). Other less common mechanisms of macrolide resistance are associated with mutations in the 23S rRNA gene sequence and/or alterations in riboproteins L4 and L22 [5]. Furthermore, an association between erythromycin resistance and cell invasiveness has been observed [6].

The major factor thought to be influencing the prevalence of macrolide resistance is macrolide consumption [7]. In addition, GAS clones showing emm types strongly associated with erythromycin resistance may contribute to the overall prevalence of macrolide resistance [8]. In this respect, Italy has always been highly ranked in the list of countries for macrolide resistance rates, ranging between $16 \%$ and $36 \%$ in Central Italy [9-11].

The aim of the present study was to examine the prevalence and phenotypic and genotypic characteristics of macrolide resistance in pharyngeal GAS isolates causing pharyngitis collected from children during two respiratory seasons (2012 and 2013) in Central Italy and compare our results with data published worldwide. Antimicrobial resistance phenotypes and genotypes were defined; the overall genotypes of strains were determined by emm typing and pulsed-field gel electrophoresis (PFGE).

\section{Materials and methods}

\section{Bacterial isolates}

GAS strains were collected from symptomatic individuals with pharyngotonsillitis from three different hospitals located in Central Italy during the respiratory season (January to June) in the years 2012 and 2013. One center is the largest referral pediatric hospital situated in Rome, while the others are general hospitals placed in Macerata and Perugia (Central Italy). All non-duplicate pharyngeal GAS clinical isolates included in the study were identified by colony morphology, $\beta$ - hemolysis on blood agar, latex agglutination test (Streptococcal Grouping Kit, Oxoid, Basingstoke, UK), and susceptibility to bacitracin disks (10 U, Oxoid, Basingstoke, UK).

\section{Antimicrobial susceptibility testing}

Antimicrobial susceptibility was determined by disk diffusion on Mueller-Hinton agar supplemented with $5 \%$ defibrinated horse blood and $20 \mathrm{mg} / \mathrm{L} \beta-\mathrm{NAD}$, according to the guidelines and interpretative criteria of the European Committee on Antimicrobial Susceptibility Testing (EUCAST). The following antibiotic disks were included (Oxoid, Basingstoke, UK): penicillin $G$, erythromycin, clindamycin, tetracycline, norfloxacin, rifampicin, quinupristin/dalfopristin, and linezolid. Norfloxacin disks were used to screen for generic fluoroquinolone resistance. Isolates categorized as norfloxacinsusceptible were considered susceptible to all fluoroquinolones. The double-disk diffusion test was used to assign the strain to either the constitutive macrolidelincosamide-streptogramin $\mathrm{B}\left(\mathrm{cMLS}_{\mathrm{B}}\right)$, the inducible $\mathrm{MLS}_{\mathrm{B}}$ (iMLS ${ }_{\mathrm{B}}$ ), or the M phenotype [10].

\section{Detection of genetic determinants of antimicrobial resistance}

Total bacterial DNA was extracted by the GenElute ${ }^{\mathrm{TM}}$ Bacterial Genomic DNA Kit (Sigma-Aldrich, St. Louis, MO, USA). Erythromycin-resistant isolates were studied for the presence of the macrolide resistance genes $\operatorname{erm}(\mathrm{A})$ [subclass $\operatorname{erm}(\mathrm{TR})], \operatorname{erm}(\mathrm{B})$, and $m e f(\mathrm{~A})$ by polymerase chain reaction (PCR) [12]. Erythromycin-resistant GAS being resistant also to tetracycline were analyzed by PCR to determine the presence of the resistance genes $\operatorname{tet}(\mathrm{M})$ and $\operatorname{tet}(\mathrm{O})$ [13].

\section{emm typing}

Isolates were emm typed according to protocols and recommendations by the Streptococcus Laboratory at the Centers for Disease Control and Prevention (CDC, Atlanta, GA, USA). Assignment of the emm type is achieved by the comparison of the query sequence with the emm type reference database. The analysis considers 90 bases encoding the N-terminal part of the mature $\mathrm{M}$ protein.

\section{PFGE macrorestriction analysis}

Total DNA from all erythromycin-resistant and a selected subgroup of erythromycin-susceptible isolates was extracted and digested with $20 \mathrm{U}$ of SmaI (Fermentas, Vilnius, Lithuania), as previously described [12]. As for DNA of isolates not digested with SmaI, the restriction enzyme Cfr9I, an isoschizomer of SmaI, was used, as previously described [14]. DNA bands 
were resolved and interpreted according to previously reported criteria [15]. Briefly, isolates with identical profiles were assigned to the same PFGE type; isolates with profiles differing by 1 to 6 bands were assigned to different subtypes within the same PFGE type; isolates with profiles showing more than 6 bands of difference were considered unrelated.

\section{Statistical analysis}

Statistical analysis was performed by Centurion XV software (STATGRAPHICS). Comparison of partitions and pairwise agreement measures were done according to Silva-Costa et al. [8] and expressed as adjusted Wallace coefficients.

\section{Results}

\section{Antibiotic resistance rates, phenotypes, and genotypes}

A total of 592 GAS isolates were collected from children with pharyngitis during the periods January-June 2012 and 2013. Patients were aged between 2 and 13 years (mean $=6.7$ years, standard deviation $=2.7$ years), and $56 \%$ were males. A total of 44 GAS were erythromycin-resistant, with an overall rate of erythromycin resistance of $7.4 \%$. Over the 2-year period under investigation, a decreasing trend of erythromycin resistance was observed (28/293 isolates, $9.6 \%$, in 2012 vs. 16/ 299, $5.4 \%$, in 2013; $p=0.06$ ).

All resistant GAS were uniformly susceptible to all antibiotics tested except for clindamycin $(52.3 \%)$ and tetracycline (50\%). All macrolide-resistant isolates with the M phenotype carried mef(A), all three isolates presenting the iMLS $\mathrm{S}_{\mathrm{B}}$ phenotype carried erm(A), and all erm(B)-positive isolates exhibited the $\mathrm{cMLS}_{\mathrm{B}}$ phenotype (Table 1). No isolates simultaneously carried more than one resistance determinant. The macrolide resistance $\mathrm{cMLS}_{\mathrm{B}} /$ erm $(\mathrm{B})$ phenotype/genotype was the most frequent $(52.3 \%)$, followed by $\mathrm{M} / \operatorname{mef}(\mathrm{A})(40.9 \%)$ and $\mathrm{iMLS}_{\mathrm{B}} / \operatorname{erm}(\mathrm{A})(6.8 \%)$ (Table 1). The prevalence of macrolide resistance phenotypes/genotypes varied slightly during the study period, with a decrement of $\mathrm{M} / m e f(\mathrm{~A})$ from 2012 to 2013, the appearance of $\mathrm{iMLS}_{\mathrm{B}} / \operatorname{erm}(\mathrm{A})$ during 2013, while $\mathrm{cMLS}_{\mathrm{B}} / \operatorname{erm}(\mathrm{B})$ remained quite constant and was the most prevalent over time (Table 1).

\section{Clonal characterization}

Among all GAS isolates collected during the 2-year survey, 25 different emm types were identified. emm types $12,89,1$, and 4 were the most prevalent, accounting for about $50 \%$ of all isolates. We found that erythromycin-resistant isolates represented only $13 \%, 2.9 \%, 4.6 \%$, and $16.7 \%$ of emm type 12 , 89,1 , and 4 subgroups, respectively. The clonal characterization of erythromycin-resistant strains is illustrated in Table 2, showing 11 different emm types, eight of which represented by two or more erythromycin-resistant strains. The predominant emm types were emm 12 and 4 (11 strains each, $25 \%$ ), followed by emm 11 (seven isolates), which were recovered in both study years and accounted for $66 \%$ of all erythromycinresistant strains (Table 2). As indicated in Table 2, the relative frequencies of emm types encountered during the two study years differed slightly, although not significantly as per Fisher's exact test analysis performed on the three most prevalent emm types. Five emm types were found during both years, while six were single year-associated but represented the less frequent $\mathrm{emm}$ types among erythromycin-resistant isolates (Table 2). In order to assess seasonal variation in macrolide-resistant emm types distribution, we divided each year into two seasonal periods (January-March and AprilJune), but no significant differences were found in the prevalence of each emm type (data not shown).

Tetracycline-resistant isolates that carried erm(B) and tet(M) belonged to five different emm types, the majority of isolates belonging to emm types 12 and 11 (seven isolates each). Three mef(A)- and tet( $(\mathrm{O})$-positive isolates were $\mathrm{emm}$ type 2, and the two isolates harboring $\operatorname{erm}(\mathrm{A})$ and $\operatorname{tet}(\mathrm{O})$ were emm type 77 (Table 2).

PFGE analysis was able to discriminate 12 different types (Table 2). An overall concordance was found between emm types and PFGE types (adjusted Wallace coefficient: 0.852; $95 \%$ confidence interval: $0.714-0.991$ ), with each emm type being associated with a specific and unique PFGE type, except for the emm type 4 group, wherein two different PFGE types were found (Table 2). Also, emm type 12, which was the most heterogeneous in terms of macrolide resistance determinants, was associated with a single PFGE type (type 3) (Table 2). It was possible to recognize three major clones, which were represented by more than three isolates and defined by the association of emm type, PFGE type, and resistance gene
Table 1 Annual and total prevalence of macrolide resistance genes and phenotypes within the 44 macrolide-resistant group A streptococcus (GAS)

\begin{tabular}{llll}
\hline Macrolide resistance gene/phenotype & \multicolumn{2}{l}{ No. of resistant strains $(\%)$} \\
\cline { 2 - 4 } & 2012 & 2013 & $2012+2013$ \\
\hline$m e f(\mathrm{~A}) / \mathrm{M}$ & $14(50 \%)$ & $4(25 \%)$ & $18(40.9 \%)$ \\
$\operatorname{erm}(\mathrm{A}) / \mathrm{iMLS} \mathrm{B}_{\mathrm{B}}$ & 0 & $3(18.75 \%)$ & $3(6.8 \%)$ \\
$\operatorname{erm}(\mathrm{B}) / \mathrm{cMLS}_{\mathrm{B}}$ & $14(50 \%)$ & $9(56.25 \%)$ & $23(52.3 \%)$ \\
\hline
\end{tabular}


Table 2 Cross tabulation of emm types, pulsed-field gel electrophoresis (PFGE) clustering, and genotypes/phenotypes of resistance for the 44 macrolide-resistant group A streptococcus (GAS) strains isolated in Italy (2012-2013)

\begin{tabular}{|c|c|c|c|c|c|c|c|c|c|c|}
\hline \multirow[t]{2}{*}{ emm type } & \multirow{2}{*}{$\begin{array}{l}\text { Percentage of susceptible } \\
\text { plus resistant isolates } \\
\text { within the general } \\
\text { population }(n=592)\end{array}$} & \multicolumn{3}{|c|}{$\begin{array}{l}\text { No. of macrolide-resistant } \\
\text { isolates per year }(\%)\end{array}$} & \multirow[t]{2}{*}{$\begin{array}{l}\text { PFGE type } \\
\text { (no. of isolates) }^{a}\end{array}$} & \multicolumn{3}{|c|}{$\begin{array}{l}\text { No. of isolates with } \\
\text { macrolide resistance gene }\end{array}$} & \multirow{2}{*}{$\begin{array}{l}\text { Tetracycline } \\
\text { resistance gene } \\
\text { (no. of isolates) }\end{array}$} & \multirow{2}{*}{$\begin{array}{l}\text { Antimicrobial } \\
\text { resistance profile } \\
\text { (no. of isolates) }\end{array}$} \\
\hline & & All & 2012 & 2013 & & $\operatorname{erm}(\mathrm{A})$ & $\operatorname{erm}(\mathrm{B})$ & $m e f(\mathrm{~A})$ & & \\
\hline 4 & 11.2 & $11(25)$ & $9(25)$ & $2(12.5)$ & $1(9) ; 2$ (2) & 0 & 0 & 11 & none & Ery (11) \\
\hline 12 & 13.0 & $11(25)$ & $7(32.1)$ & $4(25)$ & $3(11)$ & 1 & 7 & 3 & $\operatorname{tet}(\mathrm{M})(7)$ & Ery,Cli,Tet (7); Ery (4) \\
\hline 11 & 1.4 & $7(15.9)$ & $4(14.3)$ & $3(18.8)$ & $4(7)$ & 0 & 7 & 0 & $\operatorname{tet}(\mathrm{M})(7)$ & Ery,Cli,Tet (7) \\
\hline 1 & 11.2 & $3(6.8)$ & $3(10.7)$ & 0 & $5(3)$ & 0 & 3 & 0 & none & Ery,Cli (3) \\
\hline 2 & 1.2 & $3(6.8)$ & $2(7.1)$ & $1(6.3)$ & $6(3)$ & 0 & 0 & 3 & $\operatorname{tet}(\mathrm{O})(3)$ & Ery,Tet (3) \\
\hline 44 & 5.1 & $2(4.5)$ & 0 & $2(12.5)$ & $7(2)$ & 0 & 2 & 0 & $\operatorname{tet}(\mathrm{M})(2)$ & Ery,Cli,Tet (2) \\
\hline 77 & 0.3 & $2(4.5)$ & 0 & $2(12.5)$ & $8(2)$ & 2 & 0 & 0 & $\operatorname{tet}(\mathrm{O})(2)$ & Ery,Tet (2) \\
\hline 89 & 11.8 & $2(4.5)$ & $1(3.6)$ & $1(6.3)$ & $9(2)$ & 0 & 2 & 0 & $\operatorname{tet}(\mathrm{M})(1)$ & Ery,Cli,Tet (1); Ery,Cli (1) \\
\hline 18 & 3.6 & $1(2.3)$ & $1(3.6)$ & & $10(1)$ & 0 & 1 & 0 & none & Ery,Cli (1) \\
\hline 75 & 2.2 & $1(2.3)$ & $1(3.6)$ & & $11(1)$ & 0 & 0 & 1 & none & Ery (1) \\
\hline 132 & 0.2 & $1(2.3)$ & 0 & $1(6.3)$ & $12(1)$ & 0 & 1 & 0 & $\operatorname{tet}(\mathrm{M})(1)^{\mathrm{b}}$ & Ery,Cli $(1)^{\mathrm{b}}$ \\
\hline
\end{tabular}

${ }^{\text {a }}$ All isolates positive to $m e f(\mathrm{~A})$ but the emm 2 and one of the emm 12 isolates were genotyped by PFGE using $C f r 9$ I restriction enzyme, because their genomic DNA was not digested by SmaI

${ }^{\mathrm{b}}$ Isolate found to be $\operatorname{tet}(\mathrm{M})$-positive but tetracycline-susceptible

profile (Table 2). Macrolide-sensitive isolates belonging to emm types $4,11,12$, and 89 were randomly chosen and genotyped by PFGE (three isolates for each emm type). Their profiles were related to those of the most prevalent macrolideresistant strains of the corresponding $\mathrm{emm}$ types, namely PFGE type 1 for emm type 4, PFGE type 3 for emm type 12, PFGE type 4 for emm type 11, and PFGE type 9 for emm type 89 (data not shown).

\section{Discussion}

The macrolide resistance rates vary considerably among GAS strains from different countries and over time between $<3 \%$ to $>26 \%$ [2, 16-20]. In Europe from 2005 onwards, while in some regions macrolide resistance rates continued to remain high with an increasing trend, such as Greece [20], in others, such as Spain, Portugal, France, and Germany, a significant decrease of macrolide resistance rates was reported [14, 21-23].

In Italy, based on regional studies mainly, macrolide resistance rates steadily increased from $9 \%$ in 1992 to $53 \%$ in 1997 [24]. Over the period 2000-2009, the rates continued to remain high in Central Italy, varying between $16 \%$ and $36 \%$ $[10,11]$. In those years, Italy was among the regions with the highest levels of erythromycin resistance in Europe. According to the present study, we witnessed, for the first time, a decline in macrolide resistance rates in Central Italy, among GAS isolates over the period 2012-2013, down to 7.4 \%.

$\operatorname{erm}(\mathrm{B})$ was the predominant macrolide resistance gene found, followed by mef(A). erm(B) was responsible for the increase in macrolide resistance rates observed in Italy during the period 1992-1997 and remained prevalent between 2000 and $2003[10,24]$. In the present study, the erm(A) gene was rarely found, as also reported in Belgium [25].

In this study, among erythromycin-resistant isolates, emm types 12,4 , and 11 predominated, accounting for $66 \%$ of all resistant strains. In Italy, while emm types 12 and 4 were the most prevalent types among erythromycin-resistant isolates in previous studies, emm 11 was only rarely found, even among susceptible strains [10, 26]. Associations between emm types and macrolide resistance genes resulted to be the same as to those found previously, with only rare exceptions, thus suggesting that, in Italy, few successful clones are associated with macrolide resistance $[9,10,26]$. With only one exception, represented by emm type 4 with two different PFGE types, each emm type was specifically associated with a distinct PFGE type. The most prevalent emm types found among our GAS strains represent also those types frequently detected in different geographical areas [2, 20-22]. An mef(A)-positive/ emm 4 clone has been frequently found associated with macrolide resistance in GAS [8, 20, 22], as well as an erm(B)-positive/emm 11 clone that seemed to increase in prevalence in some countries [14, 21, 22, 27]. The finding of specific associations between emm type and macrolide resistance genotype and the fact that some emm types are never or rarely found in resistant isolates are suggestive of the limited transfer of macrolide resistance determinants within GAS. Nevertheless, rare emm type/resistance gene associations have been found in our study. It is the case of the second most prevalent detected clone, the erm(B)-positive/emm 12/PFGE 3 , whereas emm 12 isolates resistant to macrolides have been 
previously found associated with $\operatorname{mef}(\mathrm{A})[2,21,25]$, and the case of three emm 1 isolates with erm(B), whereas emm1-resistant isolates are generally mef(A)-positive $[22,27]$. The uncommon emm types/macrolide resistance associations observed in this study could reflect circulation of different clonal lineages in this geographic area.

The finding of 17 out of $22(77.3 \%)$ erythromycin- and tetracycline-resistant isolates that carried both $\operatorname{erm}(\mathrm{B})$ and tet $(\mathrm{M})$ could suggest that these isolates may carry conjugative transposons belonging to the Tn916 family, such as Tn3872, Tn6002, Tn6003, Tn1545, and Tn2010, where erm(B) and $\operatorname{tet}(\mathrm{M})$ are genetically associated [13]. These $\operatorname{erm}(\mathrm{B}) / \operatorname{tet}(\mathrm{M})$ isolates belonged to six different emm types, the predominant being emm types 12 and 11 (seven isolates each). While the association between emm type 11 and $\operatorname{erm}(\mathrm{B}) / \operatorname{tet}(\mathrm{M})$-mediated co-resistance has already been reported [27], emm 12 isolates carrying both $\operatorname{erm}(\mathrm{B})$ and $\operatorname{tet}(\mathrm{M})$ are very rare, due to the unusual association of this emm type with erm(B), as stated above. Three emm 2 isolates had $m e f(\mathrm{~A})$ and $\operatorname{tet}(\mathrm{O})$, suggesting that they may carry the transferable chimeric prophage $\Phi \mathrm{m} 46.1$ [28]. The two erythromycin- and tetracyclineresistant isolates of emm type 77 harbored $\operatorname{erm}(\mathrm{A})$ and $\operatorname{tet}(\mathrm{O})$.

High-level macrolide consumption, especially long-acting macrolides, have been directly associated with an increase of macrolide resistance, due to the antibiotic selective pressure, which could favor the spread of specific macrolide-resistant GAS clones, with changes in macrolide resistance rates and genotypes [19]. In some countries, reduction in macrolide consumption proved to be an important factor responsible for the decline in macrolide resistance rates [22, 23, 25]. In Italy, macrolide consumption remained relatively stable during the last 15 years (1999-2013) [29], although available data refer to the general population and not to the pediatric group only. Also, the consumption of tetracyclines, which have been associated with the increase of macrolide resistance, remained stable [29]. Although we do not have information on antibiotic usage in the patients of this study, the observed decline in macrolide resistance rates seems to not correlate to general macrolide consumption. A recent study from Slovenia indicated even the opposite; that is, a boost in resistance rates among non-invasive GAS, despite a decrease in macrolide use [30]. Thus, besides the presumed association with antibiotic use, other underlying mechanisms influencing the development and spread of antibiotic-resistant GAS isolates are to be considered, such as natural fluctuations in the prevalence of resistant clones and low fitness costs of some erythromycinresistant clones [25]. We found that the major emm types in the overall GAS pharyngeal population circulating in Italy during 2012-2013 were the same as those reported in previous Italian studies, specifically emm types 4, 12, and 89, although with differences in their relative frequencies [10,26]. Some of the most frequent emm types were also the most prevalent among erythromycin-resistant strains. PFGE was not able to differentiate resistant and susceptible isolates belonging to the same emm type, and this observation seems to indicate that the decrease of macrolide resistance would not be due to a decrement of specific GAS macrolide-resistant clones within a given $\mathrm{emm}$ type.

This study documents a decline in macrolide resistance rates in Italy, where macrolide resistance has been documented to be high in the past, and it provides useful comparative data for future epidemiological studies across erythromycinresistant GAS populations.

Acknowledgments We are grateful to Prof. Prenna Manuela for her support and critical discussion.

Funding This work was supported by funds from the Italian Ministry of Education, University and Research (MIUR, grant Futuro in Ricerca number RBFR10X4YN 001 to D.P.).

Conflict of interest The authors declare that they have no conflict of interest.

Ethical approval This article does not contain any studies with human participants or animals performed by any of the authors.

Open Access This article is distributed under the terms of the Creative Commons Attribution 4.0 International License (http:// creativecommons.org/licenses/by/4.0/), which permits unrestricted use, distribution, and reproduction in any medium, provided you give appropriate credit to the original author(s) and the source, provide a link to the Creative Commons license, and indicate if changes were made.

\section{References}

1. Bisno AL, Stevens DL (2005) Streptococcus pyogenes. In: Mandell GL, Bennett JE, Dolin R (eds) Principles and practice of infectious diseases, 6th edn. Elsevier, Philadelphia, pp 2362-2379

2. Bingen E, Bidet P, Mihaila-Amrouche L, Doit C, Forcet S, Brahimi $\mathrm{N}$ et al (2004) Emergence of macrolide-resistant Streptococcus pyogenes strains in French children. Antimicrob Agents Chemother 48:3559-3562. doi:10.1128/AAC.48.9.3559-3562. 2004

3. Bozdogan B, Appelbaum PC, Kelly LM, Hoellman DB, TambicAndrasevic A, Drukalska L et al (2003) Activity of telithromycin compared with seven other agents against 1039 Streptococcus pyogenes pediatric isolates from ten centers in central and eastern Europe. Clin Microbiol Infect 9:741-745. doi:10.1046/j.14690691.2003.00598.x

4. Sutcliffe J, Tait-Kamradt A, Wondrack L (1996) Streptococcus pneumoniae and Streptococcus pyogenes resistant to macrolides but sensitive to clindamycin: a common resistance pattern mediated by an efflux system. Antimicrob Agents Chemother 40:1817-1824

5. Roberts MC (2004) Resistance to macrolide, lincosamide, streptogramin, ketolide, and oxazolidinone antibiotics. Mol Biotechnol 28:47-62. doi:10.1385/MB:28:1:47

6. Facinelli B, Spinaci C, Magi G, Giovanetti E, Varaldo PE (2001) Association between erythromycin resistance and ability to enter human respiratory cells in group A streptococci. Lancet 358:3033. doi:10.1016/S0140-6736(00)05253-3

7. Bergman M, Huikko S, Pihlajamäki M, Laippala P, Palva E, Huovinen P et al (2004) Effect of macrolide consumption on 
erythromycin resistance in Streptococcus pyogenes in Finland in 1997-2001. Clin Infect Dis 38:1251-1256. doi:10.1086/383309

8. Silva-Costa C, Friães A, Ramirez M, Melo-Cristino J; Portuguese Group for the Study of Streptococcal Infections (2012) Differences between macrolide-resistant and -susceptible Streptococcus pyogenes: importance of clonal properties in addition to antibiotic consumption. Antimicrob Agents Chemother 56:5661-5666. doi: 10.1128/AAC.01133-12

9. Lorino G, Gherardi G, Angeletti S, De Cesaris M, Graziano N, Maringhini S et al (2006) Molecular characterisation and clonal analysis of group A streptococci causing pharyngitis among paediatric patients in Palermo, Italy. Clin Microbiol Infect 12:189-192. doi:10.1111/j.1469-0691.2005.01329.x

10. Dicuonzo G, Fiscarelli E, Gherardi G, Lorino G, Battistoni F, Landi S et al (2002) Erythromycin-resistant pharyngeal isolates of Streptococcus pyogenes recovered in Italy. Antimicrob Agents Chemother 46:3987-3990. doi:10.1128/AAC.46.12.3987-3990. 2002

11. Montagnani F, Stolzuoli L, Croci L, Rizzuti C, Arena F, Zanchi A et al (2009) Erythromycin resistance in Streptococcus pyogenes and macrolide consumption in a central Italian region. Infection 37 : 353-357. doi:10.1007/s15010-008-8023-1

12. Zampaloni C, Vitali LA, Prenna M, Toscano MA, Tempera G, Ripa S (2002) Erythromycin resistance in Italian isolates of Streptococcus pyogenes and correlations with pulsed-field gel electrophoresis analysis. Microb Drug Resist 8:39-44

13. Brenciani A, Bacciaglia A, Vecchi M, Vitali LA, Varaldo PE, Giovanetti E (2007) Genetic elements carrying erm(B) in Streptococcus pyogenes and association with tet(M) tetracycline resistance gene. Antimicrob Agents Chemother 51:1209-1216. doi:10.1128/AAC.01484-06

14. Silva-Costa C, Pinto FR, Ramirez M, Melo-Cristino J; Portuguese Surveillance Group for the Study of Respiratory Pathogens (2008) Decrease in macrolide resistance and clonal instability among Streptococcus pyogenes in Portugal. Clin Microbiol Infect 14: 1152-1159. doi:10.1111/j.1469-0691.2008.02104.x

15. Tenover FC, Arbeit RD, Goering RV, Mickelsen PA, Murray BE, Persing DH et al (1995) Interpreting chromosomal DNA restriction patterns produced by pulsed-field gel electrophoresis: criteria for bacterial strain typing. J Clin Microbiol 33:2233-2239

16. Villaseñor-Sierra A, Katahira E, Jaramillo-Valdivia AN, BarajasGarcía M de los A, Bryant A, Morfín-Otero R et al (2012) Phenotypes and genotypes of erythromycin-resistant Streptococcus pyogenes strains isolated from invasive and noninvasive infections from Mexico and the USA during 1999-2010. Int J Infect Dis 16:e178-e181. doi:10.1016/j.ijid.2011.11.005

17. Nir-Paz R, Block C, Shasha D, Korenman Z, Gorodnitzky Z, Jaffe J et al (2006) Macrolide, lincosamide and tetracycline susceptibility and emm characterisation of invasive Streptococcus pyogenes isolates in Israel. Int J Antimicrob Agents 28:313-319. doi:10.1016/j. ijantimicag.2006.07.005

18. Littauer P, Caugant DA, Sangvik M, Høiby EA, Sundsfjord A, Simonsen GS (2006) Macrolide-resistant Streptococcus pyogenes in Norway: population structure and resistance determinants. Antimicrob Agents Chemother 50:1896-1899. doi:10.1128/AAC. 50.5.1896-1899.2006
19. Silva-Costa C, Ramirez M, Melo-Cristino J; Portuguese Surveillance Group for the Study of Respiratory Pathogens (2005) Rapid inversion of the prevalences of macrolide resistance phenotypes paralleled by a diversification of $\mathrm{T}$ and emm types among Streptococcus pyogenes in Portugal. Antimicrob Agents Chemother 49:2109-2111. doi:10.1128/AAC.49.5.2109-2111. 2005

20. Michos AG, Bakoula CG, Braoudaki M, Koutouzi FI, Roma ES, Pangalis A et al (2009) Macrolide resistance in Streptococcus pyogenes: prevalence, resistance determinants, and emm types. Diagn Microbiol Infect Dis 64:295-299. doi:10.1016/j. diagmicrobio.2009.03.004

21. Montes M, Tamayo E, Mojica C, García-Arenzana JM, Esnal O, Pérez-Trallero E (2014) What causes decreased erythromycin resistance in Streptococcus pyogenes? Dynamics of four clones in a southern European region from 2005 to 2012. J Antimicrob Chemother 69:1474-1482. doi:10.1093/jac/dku039

22. d'Humières C, Cohen R, Levy C, Bidet P, Thollot F, Wollner A et al (2012) Decline in macrolide-resistant Streptococcus pyogenes isolates from French children. Int J Med Microbiol 302:300-303. doi: 10.1016/j.ijmm.2012.09.001

23. Farmand S, Henneke P, Hufnagel M, Berner R (2012) Significant decline in the erythromycin resistance of group A streptococcus isolates at a German paediatric tertiary care centre. Eur J Clin Microbiol Infect Dis 31:707-710. doi:10.1007/s10096-011-1362-3

24. Cresti S, Lattanzi M, Zanchi A, Montagnani F, Pollini S, Cellesi C et al (2002) Resistance determinants and clonal diversity in group A streptococci collected during a period of increasing macrolide resistance. Antimicrob Agents Chemother 46:1816-1822. doi:10.1128/ AAC.46.6.1816-1822.2002

25. Van Heirstraeten L, Coenen S, Lammens C, Hens N, Goossens H, Malhotra-Kumar S (2012) Antimicrobial drug use and macrolideresistant Streptococcus pyogenes, Belgium. Emerg Infect Dis 18: 1515-1518. doi:10.3201/eid1809.120049

26. Zampaloni C, Cappelletti P, Prenna M, Vitali LA, Ripa S (2003) emm Gene distribution among erythromycin-resistant and susceptible Italian isolates of Streptococcus pyogenes. J Clin Microbiol 41:1307-1310. doi:10.1128/JCM.41.3.1307-1310.2003

27. Ardanuy C, Domenech A, Rolo D, Calatayud L, Tubau F, Ayats J et al (2010) Molecular characterization of macrolide- and multidrug-resistant Streptococcus pyogenes isolated from adult patients in Barcelona, Spain (1993-2008). J Antimicrob Chemother 65:634-643. doi:10.1093/jac/dkq006

28. Di Luca MC, D’Ercole S, Petrelli D, Prenna M, Ripa S, Vitali LA (2010) Lysogenic transfer of $\operatorname{mef}(\mathrm{A})$ and tet(O) genes carried by Phim46.1 among group A streptococci. Antimicrob Agents Chemother 54:4464-4466. doi:10.1128/AAC.01318-09

29. European Centre for Disease Prevention and Control (ECDC). Trend of antimicrobial consumption by country. Available online at: http://www.ecdc.europa.eu/en/healthtopics/antimicrobial_ resistance/esac-net-database/Pages/trend-consumption-by-country. aspx. Accessed 18 March 2015

30. Cizman M, Beović B, Seme K, Paragi M, Strumbelj I, MüllerPremru $M$ et al (2006) Macrolide resistance rates in respiratory pathogens in Slovenia following reduced macrolide use. Int J Antimicrob Agents 28:537-542. doi:10.1016/j.ijantimicag.2006. 07.023 